Omale, A.P., Lorenzo, J.M., AlDhamen, A., Clift, P.D., and Webb, A.A.G., 2020, Fault

kinematics: A record of tectono-climatically controlled sedimentation along passive margins, an example from the U.S. Gulf of Mexico: GSA Bulletin, https://doi.org/10.1130/B35623.1.

\title{
Supplemental Material
}

\section{SUPPLEMENTAL FIGURE CAPTIONS}

Figure S1. Well log cross section B-B' (Bebout and Gutiérrez, 1982), one of the six cross sections, A-A' through F-F' (Fig. 3) covering southwest Louisiana, from which we measure fault displacement (Fig. 6, Tables S1-S6). Cross sections contain well logs from selected representative wells (numbered 1-12) used to identify the major horizons by lithostratigraphic and biostratigraphic correlation. Well logs are spontaneous potential (left side of each well $\log$ ) and electrical resistivity (right side of each well) logs. Faults (solid black near-vertical lines) are representative of several others in the area (Bebout and Gutiérrez, 1982), and record displacement of the tops (connected solid and dashed lines across wells) i.e., vertical separation of the major stratigraphic units (upper case letters between well logs, Fig. 5) in southwest Louisiana. Incremental throw is calculated from the fault offsets measured from well logs i.e., from the cumulative throw using the method in Figure 4. Stratigraphic units are sediments of major transgressive and regressive cycles. Biostratigraphic markers (mixed scale letters e.g. $\mathrm{N} \mathrm{s}$ ) define the relative ages of stratigraphic intervals.

Note that well $\log$ data shallower than $3000 \mathrm{ft}(\sim 0.9 \mathrm{~km})$ are absent, and that this results in limited fault displacement measurements for Upper Miocene-Recent sediments (Tables S1-S6) from the well-log cross sections. In addition, towards the south (right side of cross section), the tops of the major stratigraphic units are buried deeper than the maximum depth of the well logs and fault displacement estimates for these deeply buried sediments and their corresponding periods are unavailable.

Figure S2. Well log cross section N-N' (Bebout and Gutiérrez, 1983), one of the five cross sections M-M' through N-N' (Fig. 3), covering southeast Louisiana, from which we measure fault displacement (Fig. 7, Tables S7-S11). Cross sections contain well logs from selected representative wells (numbered 1-17) used to identify the major horizons by lithostratigraphic and biostratigraphic correlation. Well logs are spontaneous potential (left side of each well $\log$ ) and electrical resistivity (right side of each well) logs. Faults (solid black near-vertical lines) are representative of several others in the area (Bebout and Gutiérrez, 1983), and record displacement of the tops (connected solid and dashed lines across wells) i.e., vertical separation of the major stratigraphic units (upper case letters between well logs, Fig. 5) in southeast Louisiana. Incremental throw is calculated from the fault offsets measured from well logs i.e., from the cumulative throw using the method in Figure 4. Stratigraphic units are sediments of major transgressive and regressive cycles. Biostratigraphic markers (mixed scale letters e.g. Bi f) define the relative ages of stratigraphic intervals. 
Note that well log data shallower than $3000 \mathrm{ft}(\sim 0.9 \mathrm{~km})$ are absent, and that this results in limited fault displacement measurements for Upper Miocene-Recent sediments (Tables S7-S11) from the well-log cross sections. In addition, towards the south (right side of cross section), the tops of the major stratigraphic units are buried deeper than the maximum depth of the well logs and fault displacement estimates for these deeply buried sediments and their corresponding periods are unavailable.

Figure S3. Faults within seismic cross section 1 (Figure 3) from which fault throw were measured. Incremental throw is calculated from the throw cumulative throw measured directly from the seismic profile using the method in Figure 4.

Figure S4. LiDAR Digital Elevation Model (DEM) (http://atlas.lsu.edu/lidar) of southeast Louisiana, from which vertical displacement and slopes of fault scarps (Fig. 9) were measured.

\section{SUPPLEMENTAL TABLE CAPTIONS}

Table S1. Incremental Throw $(\Delta \mathrm{T})$ for cross section A-A'

Table S2. Incremental Throw $(\Delta \mathrm{T})$ for cross section B-B' Table S3. Incremental Throw $(\Delta \mathrm{T})$ for cross section C-C' Table S4. Incremental Throw $(\Delta \mathrm{T})$ for cross section D-D' Table S5. Incremental Throw $(\Delta \mathrm{T})$ for cross section E-E' Table S6. Incremental Throw $(\Delta \mathrm{T})$ for cross section F-F' Table S7. Incremental Throw $(\Delta \mathrm{T})$ for cross section M-M' Table S8. Incremental Throw $(\Delta \mathrm{T})$ for cross section N-N' Table S9. Incremental Throw $(\Delta \mathrm{T})$ for cross section O-O' Table S10. Incremental Throw $(\Delta T)$ for cross section P-P' Table S11. Incremental Throw $(\Delta \mathrm{T})$ for cross section Q-Q' Table S12. Incremental Throw $(\Delta \mathrm{T})$ for fault 1 from seismic section 1 Table S13. Incremental Throw $(\Delta \mathrm{T})$ for fault 2 from seismic section 1 Table S14. Incremental Throw $(\Delta \mathrm{T})$ for fault 3 from seismic section 1 Table S15. Incremental Throw $(\Delta \mathrm{T})$ for fault 4 from seismic section 2 Table S16. Incremental Throw $(\Delta \mathrm{T})$ for fault 5 from seismic section 3 Table S17. Incremental Throw $(\Delta \mathrm{T})$ for fault 6 from seismic section 4 Table S18. Heights and slopes of fault scarps of the Tepetate fault system (southwest Louisiana) Table S19. Heights and slopes of fault scarps of the Baton Rouge fault system (southeast Louisiana)

\section{REFERENCES CITED}

Bebout, D.G., and Gutiérrez, D.R., 1982, Regional Cross Sections, Louisiana Gulf Coast: Western Part, Louisiana Geological Survey Folio Series No. 5, 11p.

Bebout, D.G., and Gutiérrez, D.R., 1983, Regional Cross Sections, Louisiana Gulf Coast: Eastern Part, Louisiana Geological Survey Folio Series No. 6, 10p. 


\section{FIGURES S1 and S2}

Supplemental Figures 1 and 2 (well log cross sections) are the property of Louisiana Geological Survey and are available only as hard copies. These cross sections are part of the Louisiana Geological Survey Folio series no. 5 and no.6. Images of these well log cross sections were provided for review of the manuscript but cannot be published here for copyright reasons. See figure captions for more details on the well log cross sections.

For details on how to order these well log cross sections, please visit https://www.lsu.edu/lgs/publications/products/folio-series.php or contact Melissa Esnault (225578-5320) or email mesnau1@lsu.edu. 


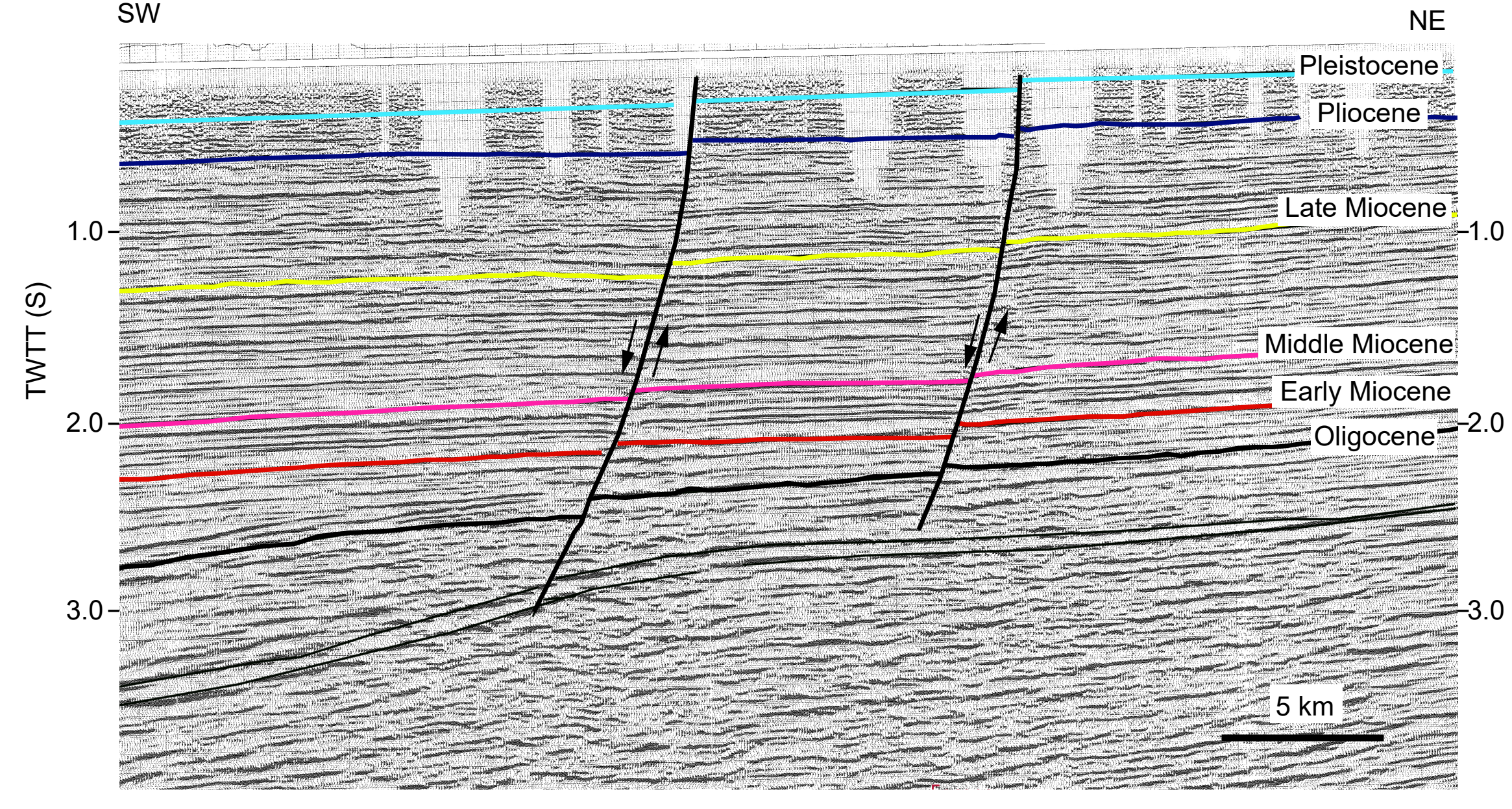

Omale_Supplemental Figure 3_Manuscript 1 
TABLE S1. INCREMENTAL THROW $(\Delta T)$ FOR CROSS SECTION A-A'

\begin{tabular}{|c|c|c|c|c|c|c|c|c|c|c|c|c|c|c|c|}
\hline \multirow[t]{2}{*}{ Unit $^{*}$} & \multicolumn{12}{|c|}{ Incremental throw (m) } & \multirow[b]{2}{*}{13} & \multirow[b]{2}{*}{14} & \multirow[b]{2}{*}{15} \\
\hline & $1^{\dagger}$ & 2 & 3 & 4 & 5 & 6 & 7 & 8 & 9 & 10 & 11 & 12 & & & \\
\hline Middle Miocene & N.D ${ }^{\S}$. & N.D. & N.D. & N.D. & N.D. & N.D. & N.D. & N.D. & N.D. & N.D. & N.D. & N.D. & N.D. & N.D. & 60 \\
\hline Lower Miocene & N.D. & N.D. & N.D. & N.D. & N.D. & N.D. & N.D. & N.D. & N.D. & 15 & 38 & 83 & 60 & 195 & 120 \\
\hline Anahuac & N.D. & N.D. & N.D. & N.D. & N.D. & N.D. & N.D. & N.D. & N.D. & 68 & 120 & 98 & 75 & 120 & N.D. \\
\hline Frio & N.D. & N.D. & N.D. & N.D. & 23 & 15 & 0 & 30 & 45 & 83 & N.D. & N.D. & N.D. & N.D. & N.D. \\
\hline Vicksburg/Jackson & N.D. & N.D. & 15 & 15 & 0 & 0 & 15 & 15 & 23 & N.D. & N.D. & N.D. & N.D. & N.D. & N.D. \\
\hline Cockfield (Yegua) & N.D. & N.D. & 15 & 23 & 0 & 8 & 45 & 45 & N.D. & N.D. & N.D. & N.D. & N.D. & N.D. & N.D. \\
\hline Sparta & 68 & 38 & 0 & 0 & 0 & 8 & 30 & 98 & N.D. & N.D. & N.D. & N.D. & N.D. & N.D. & N.D. \\
\hline
\end{tabular}

TABLE S2. INCREMENTAL THROW $(\Delta T)$ FOR CROSS SECTION B-B'

\begin{tabular}{|c|c|c|c|c|c|c|c|c|c|c|c|}
\hline \multirow[t]{2}{*}{ Unit $^{*}$} & \multicolumn{11}{|c|}{ Incremental throw (m) } \\
\hline & $1^{\dagger}$ & 2 & 3 & 4 & 5 & 6 & 7 & 8 & 9 & 10 & 11 \\
\hline Middle Miocene & N.D ${ }^{\S}$. & N.D. & N.D. & N.D. & N.D. & N.D. & N.D. & N.D. & N.D. & 75 & N.D. \\
\hline Lower Miocene & N.D. & N.D. & N.D. & N.D. & N.D. & N.D. & N.D. & N.D. & 1035 & 285 & 248 \\
\hline Anahuac & N.D. & N.D. & N.D. & N.D. & N.D. & N.D. & N.D. & N.D. & 135 & N.D. & N.D. \\
\hline Frio & N.D. & N.D. & N.D. & N.D. & N.D. & N.D. & 37.5 & 540 & N.D. & N.D. & N.D. \\
\hline Vicksburg/Jackson & N.D. & N.D. & N.D. & 60 & 120 & 135 & 15 & 240 & N.D. & N.D. & N.D. \\
\hline Cockfield (Yegua) & N.D. & 15 & 30 & 7.5 & 22.5 & 150 & 7.5 & N.D. & N.D. & N.D. & N.D. \\
\hline Sparta & 30 & 75 & 22.5 & 7.5 & 7.5 & N.D. & 90 & N.D. & N.D. & N.D. & N.D. \\
\hline Wilcox & 45 & N.D. & N.D. & N.D. & N.D. & N.D. & N.D. & N.D. & N.D. & N.D. & N.D. \\
\hline Midway & 7.5 & N.D. & N.D. & N.D. & N.D. & N.D. & N.D. & N.D. & N.D. & N.D. & N.D. \\
\hline
\end{tabular}

*See figure 3 for location and stratigraphic chart in figure 5 for ages.

$\dagger$ Numbers 1-11 represent faults identified sequentially from north to south within cross section B-B'.

${ }^{\S}$ N.D. = no data. 
TABLE S3. INCREMENTAL THROW $(\Delta T)$ FOR CROSS SECTION C-C'

\begin{tabular}{|c|c|c|c|c|c|c|c|c|c|c|c|c|c|}
\hline \multirow[t]{2}{*}{ Unit* } & \multicolumn{13}{|c|}{ Incremental throw (m) } \\
\hline & $1^{\dagger}$ & 2 & 3 & 4 & 5 & 6 & 7 & 8 & 9 & 10 & 11 & 12 & 13 \\
\hline Middle Miocene & N.D ${ }^{\S}$. & N.D. & N.D. & N.D. & N.D. & N.D. & N.D. & N.D. & N.D. & N.D. & N.D. & 22.5 & 90 \\
\hline Lower Miocene & N.D. & N.D. & N.D. & N.D. & 22.5 & 15 & 30 & 90 & 60 & 150 & 375 & 52.5 & 75 \\
\hline Anahuac & 15 & N.D. & N.D. & N.D. & 30 & 7.5 & 172.5 & 0 & 75 & 30 & 345 & 15 & N.D. \\
\hline Frio & 22.5 & 15 & 15 & 7.5 & 37.5 & 60 & 217.5 & N.D. & N.D. & N.D. & N.D. & N.D. & N.D. \\
\hline Vicksburg/Jackson & 15 & 0 & 0 & 7.5 & 60 & 37.5 & N.D. & N.D. & N.D. & N.D. & N.D. & N.D. & N.D. \\
\hline Cockfield (Yegua) & 22.5 & 0 & 0 & 7.5 & 90 & 60 & N.D. & N.D. & N.D. & N.D. & N.D. & N.D. & N.D. \\
\hline Sparta & 30 & 67.5 & 45 & 67.5 & 30 & N.D. & N.D. & N.D. & N.D. & N.D. & N.D. & N.D. & N.D. \\
\hline
\end{tabular}

TABLE S4. INCREMENTAL THROW $(\Delta T)$ FOR CROSS SECTION D-D'

\begin{tabular}{|c|c|c|c|c|c|c|c|c|c|c|c|c|c|c|}
\hline \multirow[t]{2}{*}{ Unit* } & \multicolumn{14}{|c|}{ Incremental throw (m) } \\
\hline & $1^{\dagger}$ & 2 & 3 & 4 & 5 & 6 & 7 & 8 & 9 & 10 & 11 & 12 & 13 & 14 \\
\hline Upper Miocene & N.D ${ }^{\S}$. & N.D. & N.D. & N.D. & N.D. & N.D. & N.D. & N.D. & N.D. & N.D. & N.D. & 15 & N.D. & N.D. \\
\hline Middle Miocene & N.D. & N.D. & N.D. & N.D. & N.D. & N.D. & N.D. & N.D. & N.D. & 15 & 15 & 15 & 67.5 & 45 \\
\hline Lower Miocene & N.D. & N.D. & N.D. & N.D. & N.D. & 60 & 60 & 67.5 & 90 & 120 & 45 & N.D. & N.D. & N.D. \\
\hline Anahuac & N.D. & N.D. & N.D. & N.D. & N.D. & 15 & 15 & 22.5 & 75 & N.D. & N.D. & N.D. & N.D. & N.D. \\
\hline Frio & N.D. & 30 & 22.5 & N.D. & 97.5 & 45 & 75 & N.D. & N.D. & N.D. & N.D. & N.D. & N.D. & N.D. \\
\hline Vicksburg/Jackson & 30 & 7.5 & 60 & 105 & 52.5 & N.D. & N.D. & N.D. & N.D. & N.D. & N.D. & N.D. & N.D. & N.D. \\
\hline Cockfield (Yegua) & 0 & 22.5 & 37.5 & 15 & N.D. & N.D. & N.D. & N.D. & N.D. & N.D. & N.D. & N.D. & N.D. & N.D. \\
\hline Sparta & 52.5 & 45 & 0 & 60 & N.D. & N.D. & N.D. & N.D. & N.D. & N.D. & N.D. & N.D. & N.D. & N.D. \\
\hline Wilcox & N.D. & 30 & 52.5 & N.D. & N.D. & N.D. & N.D. & N.D. & N.D. & N.D. & N.D. & N.D. & N.D. & N.D. \\
\hline
\end{tabular}

*See figure 3 for location and stratigraphic chart in figure 5 for ages.

$\dagger$ Numbers 1-14 represent faults identified sequentially from north to south within cross section D-D'.

${ }^{\S}$ N.D. = no data. 
TABLE S5. INCREMENTAL THROW $(\Delta T)$ FOR CROSS SECTION E-E'

\begin{tabular}{|c|c|c|c|c|c|c|c|c|c|c|c|c|c|}
\hline \multirow[t]{2}{*}{ Unit* } & \multicolumn{13}{|c|}{ Incremental throw (m) } \\
\hline & $1^{\dagger}$ & 2 & 3 & 4 & 5 & 6 & 7 & 8 & 9 & 10 & 11 & 12 & 13 \\
\hline Middle Miocene & N.D ${ }^{\S}$. & N.D. & N.D. & N.D. & 15 & 60 & 22.5 & 30 & 150 & 45 & 75 & 30 & 30 \\
\hline Lower Miocene & N.D. & N.D. & 30 & 30 & 30 & 97.5 & 22.5 & 0 & 195 & 52.5 & 60 & 60 & N.D. \\
\hline Anahuac & 60 & N.D. & 112.5 & 15 & 7.5 & 157.5 & 120 & 345 & N.D. & 37.5 & N.D. & N.D. & N.D. \\
\hline Frio & 7.5 & 15 & 45 & 135 & 247.5 & N.D. & N.D. & N.D. & N.D. & N.D. & N.D. & N.D. & N.D. \\
\hline Vicksburg/Jackson & 15 & 30 & 247.5 & 135 & N.D. & N.D. & N.D. & N.D. & N.D. & N.D. & N.D. & N.D. & N.D. \\
\hline Cockfield (Yegua) & 37.5 & 15 & 150 & N.D. & N.D. & N.D. & N.D. & N.D. & N.D. & N.D. & N.D. & N.D. & N.D. \\
\hline Sparta & 75 & 7.5 & 15 & N.D. & N.D. & N.D. & N.D. & N.D. & N.D. & N.D. & N.D. & N.D. & N.D. \\
\hline Wilcox & 225 & N.D. & N.D. & N.D. & N.D. & N.D. & N.D. & N.D. & N.D. & N.D. & N.D. & N.D. & N.D. \\
\hline
\end{tabular}

${ }^{*}$ See figure 3 for location and stratigraphic chart in figure 5 for ages.

$\dagger$ Numbers 1-13 represent faults identified sequentially from north to south within cross section E-E'.

${ }^{\S}$ N.D. = no data.

TABLE S6. INCREMENTAL THROW $(\Delta T)$ FOR CROSS SECTION F-F'

\begin{tabular}{|c|c|c|c|c|c|c|c|c|c|c|}
\hline \multirow[t]{2}{*}{ Unit* } & \multicolumn{10}{|c|}{ Incremental throw (m) } \\
\hline & $1^{\dagger}$ & 2 & 3 & 4 & 5 & 6 & 7 & 8 & 9 & 10 \\
\hline Pliocene & N.D ${ }^{\S}$. & N.D. & N.D. & N.D. & N.D. & N.D. & N.D. & N.D. & N.D. & N.D. \\
\hline Upper Miocene & N.D. & N.D. & N.D. & N.D. & N.D. & N.D. & N.D. & N.D. & N.D. & N.D. \\
\hline Middle Miocene & N.D. & N.D. & N.D. & N.D. & N.D. & N.D. & N.D. & N.D. & N.D. & 37.5 \\
\hline Lower Miocene & N.D. & N.D. & N.D. & N.D. & 187.5 & 112.5 & 97.5 & 135 & 120 & 37.5 \\
\hline Anahuac & 15 & 0 & 15 & 0 & 22.5 & 15 & 7.5 & 30 & 15 & 52.5 \\
\hline Frio & 15 & 15 & 22.5 & 15 & 15 & 532.5 & 270 & 135 & 225 & N.D. \\
\hline Vicksburg/Jackson & 0 & 0 & 7.5 & 90 & 390 & 300 & N.D. & N.D. & N.D. & N.D. \\
\hline Cockfield (Yegua) & 22.5 & 0 & 0 & 30 & 150 & N.D. & N.D. & N.D. & N.D. & N.D. \\
\hline Sparta & 7.5 & 60 & 30 & 30 & 112.5 & N.D. & N.D. & N.D. & N.D. & N.D. \\
\hline Wilcox & 135 & N.D. & N.D. & N.D. & N.D. & N.D. & N.D. & N.D. & N.D. & N.D. \\
\hline
\end{tabular}


TABLE S6 CONTINUED. INCREMENTAL THROW $(\Delta T)$ FOR CROSS SECTION F-F'

\begin{tabular}{lcccccccccc}
\hline \hline Unit* & \multicolumn{7}{c}{ Incremental throw $(\mathrm{m})$} & 18 & 19 \\
\cline { 2 - 9 } & $11^{\dagger}$ & 12 & 13 & 14 & 15 & 16 & 17 & 18 \\
\hline Pliocene & N.D & N.D. & N.D. & N.D. & N.D. & N.D. & N.D. & N.D. & 15 \\
Upper Miocene & N.D. & N.D. & N.D. & 22.5 & N.D. & 52.5 & 75 & 97.5 & 30 & 30 \\
Middle Miocene & N.D. & N.D. & 210 & 60 & 165 & 150 & 127.5 & 67.5 & 30 & 60 \\
Lower Miocene & 37.5 & 37.5 & 570 & 97.5 & 22.5 & 270 & 270 & N.D. & N.D. & N.D. \\
Anahuac & 22.5 & 202.5 & 697.5 & N.D. & N.D. & N.D. & N.D. & N.D. & N.D. & N.D. \\
Frio & N.D. & N.D. & N.D. & N.D. & N.D. & N.D. & N.D. & N.D. & N.D. & N.D. \\
Vicksburg/Jackson & N.D. & N.D. & N.D. & N.D. & N.D. & N.D. & N.D. & N.D. & N.D. & N.D. \\
Cockfield (Yegua) & N.D. & N.D. & N.D. & N.D. & N.D. & N.D. & N.D. & N.D. & N.D. & N.D. \\
Sparta & N.D. & N.D. & N.D. & N.D. & N.D. & N.D. & N.D. & N.D. & N.D. & N.D. \\
Wilcox & N.D. & N.D. & N.D. & N.D. & N.D. & N.D. & N.D. & N.D. & N.D. & N.D. \\
\hline
\end{tabular}

*See figure 3 for location and stratigraphic chart in figure 5 for ages.

$\dagger$ Numbers 11-20 represent faults identified sequentially from north to south within cross section F-F'.

${ }^{\S}$ N.D. = no data.

TABLE S7. INCREMENTAL THROW $(\Delta \mathrm{T})$ FOR CROSS SECTION M-M'

\begin{tabular}{|c|c|c|c|c|c|c|c|c|c|c|c|c|c|c|}
\hline \multirow[t]{2}{*}{ Unit* } & \multicolumn{14}{|c|}{ Incremental throw (m) } \\
\hline & $1^{\dagger}$ & 2 & 3 & 4 & 5 & 6 & 7 & 8 & 9 & 10 & 11 & 12 & 13 & 14 \\
\hline Pliocene & N.D ${ }^{\S}$. & N.D. & N.D. & N.D. & N.D. & 67.5 & 90 & 15 & 7.5 & N.D. & 127.5 & 195 & 90 & 525 \\
\hline Upper Miocene & N.D. & N.D. & N.D. & N.D. & N.D. & 97.5 & 30 & 0 & 22.5 & 345 & 262.5 & 435 & 15 & 420 \\
\hline Middle Miocene & 30 & 30 & 52.5 & 45 & 45 & 120 & N.D. & 90 & 135 & N.D. & N.D. & N.D. & N.D. & N.D. \\
\hline Lower Miocene & 30 & 30 & 67.5 & 120 & 105 & 195 & N.D. & N.D. & N.D. & N.D. & N.D. & N.D. & N.D. & N.D. \\
\hline Anahuac & 45 & 60 & 7.5 & 45 & N.D. & N.D. & N.D. & N.D. & N.D. & N.D. & N.D. & N.D. & N.D. & N.D. \\
\hline
\end{tabular}


TABLE S8. INCREMENTAL THROW $(\Delta \mathrm{T})$ FOR CROSS SECTION N-N'

\begin{tabular}{lccccccccc}
\hline \hline Unit* & \multicolumn{7}{c}{ Incremental throw (m) } \\
\cline { 2 - 8 } & $1^{\dagger}$ & 2 & 3 & 4 & 5 & 6 & 7 & 8 \\
\hline Pliocene & N.D & N.D. & N.D. & N.D. & N.D. & 15 & 52.5 & 15 \\
Upper Miocene & N.D. & N.D. & N.D. & 30 & 30 & 15 & 37.5 & 15 \\
Middle Miocene & N.D. & 37.5 & 15 & 15 & 105 & 45 & 120 & 45 \\
Lower Miocene & 22.5 & 30 & 0 & 15 & 75 & N.D. & N.D. & N.D. & 45 \\
Anahuac & 7.5 & 90 & 15 & 60 & 150 & N.D. & N.D. & N.D. & N.D. \\
Frio & 15 & 22.5 & N.D. & N.D. & N.D. & N.D. & N.D. & N.D. & N.D. \\
Vicksburg/Jackson & 15 & 45 & N.D. & N.D. & N.D. & N.D. & N.D. & N.D. & N.D. \\
Cockfield (Yegua) & 37.5 & 30 & N.D. & N.D. & N.D. & N.D. & N.D. & N.D. & N.D. \\
Sparta & 52.5 & 75 & N.D. & N.D. & N.D. & N.D. & N.D. & N.D. & N.D. \\
\hline
\end{tabular}

*See figure 3 for location and stratigraphic chart in figure 5 for ages.

†Numbers 1-9 represent faults identified sequentially from north to south within cross section N-N'.

${ }^{\S}$ N.D. = no data.

TABLE S8 CONTINUED. INCREMENTAL THROW $(\Delta T)$ FOR CROSS SECTION N-N'

\begin{tabular}{|c|c|c|c|c|c|c|c|c|}
\hline \multirow[t]{2}{*}{ Unit* } & \multicolumn{8}{|c|}{ Incremental throw (m) } \\
\hline & $10^{\dagger}$ & 11 & 12 & 13 & 14 & 15 & 16 & 17 \\
\hline Pliocene & 30 & 15 & 45 & 15 & 60 & 105 & 90 & 67.5 \\
\hline Upper Miocene & 52.5 & 15 & 15 & N.D ${ }^{\S}$. & 75 & 375 & 180 & 172.5 \\
\hline Middle Miocene & 202.5 & 180 & N.D. & N.D. & N.D. & N.D. & N.D. & N.D. \\
\hline Lower Miocene & N.D. & N.D. & N.D. & N.D. & N.D. & N.D. & N.D. & N.D. \\
\hline Anahuac & N.D. & N.D. & N.D. & N.D. & N.D. & N.D. & N.D. & N.D. \\
\hline Frio & N.D. & N.D. & N.D. & N.D. & N.D. & N.D. & N.D. & N.D. \\
\hline Vicksburg/Jackson & N.D. & N.D. & N.D. & N.D. & N.D. & N.D. & N.D. & N.D. \\
\hline Cockfield (Yegua) & N.D. & N.D. & N.D. & N.D. & N.D. & N.D. & N.D. & N.D. \\
\hline Sparta & N.D. & N.D. & N.D. & N.D. & N.D. & N.D. & N.D. & N.D. \\
\hline
\end{tabular}

*See figure 3 for location and stratigraphic chart in figure 5 for ages.

$\dagger$ Numbers 10-17 represent faults identified sequentially from north to south within cross section N-N'.

${ }^{\S}$ N.D. = no data. 
TABLE S9. INCREMENTAL THROW $(\Delta T)$ FOR CROSS SECTION O-O'

\begin{tabular}{|c|c|c|c|c|c|c|c|}
\hline \multirow[t]{2}{*}{ Unit* } & \multicolumn{7}{|c|}{ Incremental throw (m) } \\
\hline & $1^{\dagger}$ & 2 & 3 & 4 & 5 & 6 & 7 \\
\hline Pliocene & N.D ${ }^{\S}$. & N.D. & N.D. & N.D. & N.D. & N.D. & 75 \\
\hline Upper Miocene & N.D. & N.D. & N.D. & N.D. & N.D. & 90 & 1005 \\
\hline Middle Miocene & N.D. & 60 & 120 & 75 & 405 & N.D. & N.D. \\
\hline Lower Miocene & 180 & 157.5 & 555 & N.D. & N.D. & N.D. & N.D. \\
\hline Anahuac & 0 & N.D. & N.D. & N.D. & N.D. & N.D. & N.D. \\
\hline Frio & 60 & N.D. & N.D. & N.D. & N.D. & N.D. & N.D. \\
\hline Vicksburg/Jackson & 15 & N.D. & N.D. & N.D. & N.D. & N.D. & N.D. \\
\hline Cockfield (Yegua) & 0 & N.D. & N.D. & N.D. & N.D. & N.D. & N.D. \\
\hline Sparta & 30 & N.D. & N.D. & N.D. & N.D. & N.D. & N.D. \\
\hline Wilcox & 13 & N.D. & N.D. & N.D. & N.D. & N.D. & N.D. \\
\hline
\end{tabular}

*See figure 3 for location and stratigraphic chart in figure 5 for ages.

$\dagger$ Numbers 1-7 represent faults identified sequentially from north to south within cross section O-O'.

${ }^{\S}$ N.D. = no data.

TABLE S10. INCREMENTAL THROW ( $\Delta$ T) FOR CROSS SECTION P-P'

\begin{tabular}{|c|c|c|c|c|c|c|c|c|}
\hline \multirow[t]{2}{*}{ Unit* } & \multicolumn{8}{|c|}{ Incremental throw (m) } \\
\hline & $1^{\dagger}$ & 2 & 3 & 4 & 5 & 6 & 7 & 8 \\
\hline Upper Miocene & N.D ${ }^{\S}$. & N.D. & N.D. & N.D. & N.D. & 90 & 1395 & 367.5 \\
\hline Middle Miocene & N.D. & N.D. & 360 & 202.5 & 195 & N.D. & N.D. & N.D. \\
\hline Lower Miocene & 255 & 210 & N.D. & N.D. & N.D. & N.D. & N.D. & N.D. \\
\hline Anahuac & 0 & N.D. & N.D. & N.D. & N.D. & N.D. & N.D. & N.D. \\
\hline Frio & 0 & N.D. & N.D. & N.D. & N.D. & N.D. & N.D. & N.D. \\
\hline Vicksburg/Jackson & 0 & N.D. & N.D. & N.D. & N.D. & N.D. & N.D. & N.D. \\
\hline Cockfield (Yegua) & 0 & N.D. & N.D. & N.D. & N.D. & N.D. & N.D. & N.D. \\
\hline Sparta & 15 & N.D. & N.D. & N.D. & N.D. & N.D. & N.D. & N.D. \\
\hline
\end{tabular}

*See figure 3 for location and stratigraphic chart in figure 5 for ages.

$\dagger$ Numbers 1-8 represent faults identified sequentially from north to south within cross section P-P'.

${ }^{\S}$ N.D. = no data. 
TABLE S11. INCREMENTAL THROW $(\Delta \mathrm{T})$ FOR CROSS SECTION Q-Q'

\begin{tabular}{|c|c|c|c|c|c|c|c|}
\hline \multirow[t]{2}{*}{ Unit* } & \multicolumn{7}{|c|}{ Incremental throw (m) } \\
\hline & $1^{\dagger}$ & 2 & 3 & 4 & 5 & 6 & 7 \\
\hline Upper Miocene & 0 & 0 & 0 & 0 & 0 & 60 & 450 \\
\hline Middle Miocene & 22.5 & 30 & 157.5 & 75 & 210 & N.D ${ }^{\S}$. & N.D. \\
\hline Lower Miocene & 37.5 & 60 & 22.5 & 885 & N.D. & N.D. & N.D. \\
\hline Anahuac & 15 & 30 & 120 & N.D. & N.D. & N.D. & N.D. \\
\hline Frio & 15 & 15 & 15 & N.D. & N.D. & N.D. & N.D. \\
\hline Vicksburg/Jackson & 15 & 0 & N.D. & N.D. & N.D. & N.D. & N.D. \\
\hline Cockfield (Yegua) & 15 & 0 & N.D. & N.D. & N.D. & N.D. & N.D. \\
\hline Sparta & 22.5 & 0 & N.D. & N.D. & N.D. & N.D. & N.D. \\
\hline Wilcox & 22.5 & 30 & N.D. & N.D. & N.D. & N.D. & N.D. \\
\hline Midway & 15 & 0 & N.D. & N.D. & N.D. & N.D. & N.D. \\
\hline
\end{tabular}


TABLE S12. INCREMENTAL THROW $(\triangle \mathrm{T})$ FOR FAULT 1 FROM SEISMIC SECTION 1

\begin{tabular}{lccc}
\hline Unit & Age $(\mathrm{Ma})^{*}$ & Throw $(\mathrm{m})$ & Throw Error $(\mathrm{m})$ \\
\hline Pleistocene & 0 & 38 & 5.7 \\
Pliocene & 2.59 & 38 & 5.7 \\
Late Miocene & 5.33 & 15 & 2.25 \\
Early Miocene & 11.63 & 45 & 6.75 \\
\hline
\end{tabular}

*Fault 1 is the first fault from north to south within the cross section. See figure 3 for location.

"Numbers are for the end of the period represented by each chronostratigraphic unit according to Gradstein et al., 2012.

TABLE S13. INCREMENTAL THROW ( $\Delta$ T) FOR FAULT 2 FROM SEISMIC SECTION $1 *$

\begin{tabular}{lccc}
\hline \hline Unit & Age $(\mathrm{Ma})^{\dagger}$ & Throw $(\mathrm{m})$ & Throw Error $(\mathrm{m})$ \\
\hline Pleistocene & 0 & 31 & 1 \\
Pliocene & 2.59 & 46 & 10 \\
Late Miocene & 5.33 & 30 & 7 \\
\hline
\end{tabular}

*Fault 2 is the second fault from north to south within the cross section. See figure 3 for location.

Numbers are for the end of the period represented by each chronostratigraphic unit according to Gradstein et al., 2012.

TABLE S14. INCREMENTAL THROW $(\Delta T)$ FOR FAULT 3 FROM SEISMIC SECTION $1 *$

\begin{tabular}{lccc}
\hline Unit & Age $(\mathrm{Ma})^{\dagger}$ & Throw $(\mathrm{m})$ & Throw Error $(\mathrm{m})$ \\
\hline Pleistocene & 0 & 30 & 4.5 \\
Pliocene & 2.59 & 61 & 9.15 \\
Early Miocene & 11.63 & 274 & 41.1 \\
\hline
\end{tabular}

*Fault 3 is the third fault from north to south within the cross section. See figure 3 for location.

${ }^{\dagger}$ Numbers are for the end of the period represented by each chronostragraphic unit according to Gradstein et al., 2012.

TABLE S15. INCREMENTAL THROW $(\Delta \mathrm{T})$ FOR FAULT 4 FROM SEISMIC SECTION 2*

\begin{tabular}{lccc}
\hline \hline Unit & Age $(\mathrm{Ma}))^{\dagger}$ & Throw $(\mathrm{m})$ & Throw Error $(\mathrm{m})$ \\
\hline Pleistocene & 0 & 18 & 2.7 \\
Pliocene & 2.59 & 46 & 6.9 \\
\hline "Numbers are for the end of the period represented by each chronostratigraphic unit according \\
to Gradstein et al., 2012.
\end{tabular}


TABLE S16. INCREMENTAL THROW $(\Delta \mathrm{T})$ FOR FAULT 5 FROM SEISMIC SECTION 3

\begin{tabular}{lccc}
\hline \hline Unit & Age $(\mathrm{Ma})^{*}$ & Throw $(\mathrm{m})$ & Throw Error $(\mathrm{m})$ \\
\hline Pleistocene & 0 & 34 & 5.1 \\
Pliocene & 2.59 & 58 & 8.7 \\
* Numbers are for the end of the period represented by each chronostratigraphic unit according \\
to Gradstein et al., 2012.
\end{tabular}

TABLE S17. INCREMENTAL THROW $(\Delta T)$ FOR FAULT 6 FROM SEISMIC SECTION 4

\begin{tabular}{lccc}
\hline \hline Unit & Age $(\mathrm{Ma})^{*}$ & Throw $(\mathrm{m})$ & Throw Error $(\mathrm{m})$ \\
\hline Pleistocene & 0 & 61 & 9.15 \\
Pliocene & 2.59 & 137 & 20.55 \\
Late Miocene & 5.33 & 122 & 18.3 \\
\hline "Numbers are for the end of the period represented by each chronostratigraphic unit according \\
to Gradstein et al., 2012.
\end{tabular}


TABLE S18. HEIGHTS AND SLOPES OF FAULT SCARPS OF THE TEPETATE FAULT SYSTEM (SOUTHWEST LOUISIANA)

\begin{tabular}{|c|c|c|c|c|c|}
\hline \multicolumn{2}{|c|}{ Location } & \multirow[b]{2}{*}{ Height (m) } & \multirow[b]{2}{*}{ Slope $(\mathrm{m} / \mathrm{m})$} & \multirow[b]{2}{*}{$\begin{array}{l}\text { Height Error } \\
(\mathrm{m})\end{array}$} & \multirow[b]{2}{*}{$\begin{array}{c}\text { Slope Error } \\
(\mathrm{m})\end{array}$} \\
\hline Longitude & Latitude & & & & \\
\hline-92.86622 & 30.42688 & 1.779 & -0.0050 & 0.004 & 0.00001057 \\
\hline-92.84145 & 30.42346 & 2.011 & -0.0102 & 0.028 & 0.00003497 \\
\hline-92.85756 & 30.42509 & 2.321 & -0.0118 & 0.010 & 0.00006402 \\
\hline-92.85124 & 30.42457 & 2.366 & -0.0173 & 0.046 & 0.00000177 \\
\hline-92.83753 & 30.42189 & 1.296 & -0.0088 & 0.015 & 0.00015851 \\
\hline-92.82443 & 30.42138 & 3.364 & -0.0076 & 0.015 & 0.00006472 \\
\hline-92.83069 & 30.42177 & 2.635 & -0.0089 & 0.018 & 0.00001935 \\
\hline-92.78403 & 30.41658 & 1.903 & -0.0075 & 0.010 & 0.00001134 \\
\hline-92.79676 & 30.42068 & 1.800 & -0.0112 & 0.016 & 0.00006655 \\
\hline-92.76364 & 30.41910 & 2.238 & -0.0063 & 0.014 & 0.00007796 \\
\hline-92.74158 & 30.42528 & 1.449 & -0.0155 & 0.019 & 0.00006817 \\
\hline-92.73450 & 30.42552 & 2.575 & -0.0174 & 0.017 & 0.00000350 \\
\hline-92.71548 & 30.42631 & 2.882 & -0.0228 & 0.020 & 0.00013765 \\
\hline-92.72113 & 30.42583 & 3.055 & -0.0224 & 0.030 & 0.00038350 \\
\hline-92.70175 & 30.42791 & 4.065 & -0.0183 & 0.014 & 0.00000237 \\
\hline-92.65513 & 30.43283 & 1.367 & -0.0069 & 0.007 & 0.00000029 \\
\hline-92.68573 & 30.43306 & 2.053 & -0.0046 & 0.048 & 0.00004259 \\
\hline-92.74718 & 30.42475 & 1.425 & -0.0129 & 0.015 & 0.00002633 \\
\hline-93.52053 & 30.38733 & 4.264 & -0.0168 & 0.016 & 0.00021559 \\
\hline-93.51889 & 30.38835 & 5.041 & -0.0114 & 0.022 & 0.00001602 \\
\hline-93.51618 & 30.38940 & 7.724 & -0.0174 & 0.064 & 0.00063379 \\
\hline-93.51375 & 30.38892 & 7.399 & -0.0167 & 0.012 & 0.00003615 \\
\hline-93.50662 & 30.39156 & 8.781 & -0.0149 & 0.030 & 0.00047771 \\
\hline-93.49750 & 30.39596 & 3.171 & -0.0072 & 0.012 & 0.00000891 \\
\hline-93.49103 & 30.39946 & 5.684 & -0.0160 & 0.019 & 0.00039537 \\
\hline-93.48251 & 30.40265 & 4.055 & -0.0183 & 0.010 & 0.00000002 \\
\hline-93.47807 & 30.40433 & 1.765 & -0.0090 & 0.009 & 0.00001609 \\
\hline-93.47605 & 30.40516 & 1.345 & -0.0053 & 0.015 & 0.00000141 \\
\hline-93.47372 & 30.40630 & 1.179 & -0.0027 & 0.011 & 0.00005461 \\
\hline-93.46657 & 30.40955 & 4.538 & -0.0179 & 0.035 & 0.00057957 \\
\hline-93.45913 & 30.41211 & 7.652 & -0.0216 & 0.207 & 0.00021344 \\
\hline-93.45009 & 30.41313 & 12.696 & -0.0430 & 0.051 & 0.00000352 \\
\hline-93.44764 & 30.41330 & 9.183 & -0.0363 & 0.237 & 0.00287099 \\
\hline-93.43764 & 30.41690 & 5.772 & -0.0391 & 0.091 & 0.00007633 \\
\hline-93.43161 & 30.41760 & 1.016 & -0.0189 & 0.014 & 0.00012397 \\
\hline-93.42495 & 30.41925 & 0.650 & -0.0165 & 0.650 & 0.00017092 \\
\hline-93.41610 & 30.42164 & 4.060 & -0.0092 & 0.030 & 0.00001164 \\
\hline-93.41195 & 30.42087 & 8.138 & -0.0275 & 0.021 & 0.00025372 \\
\hline-93.40769 & 30.42061 & 10.850 & -0.0367 & 0.041 & 0.00136682 \\
\hline-93.40281 & 30.41968 & 6.446 & -0.0109 & 0.026 & 0.00000000 \\
\hline-93.39957 & 30.42095 & 11.308 & -0.0255 & 0.054 & 0.00111207 \\
\hline
\end{tabular}


TABLE S18 CONTINUED. HEIGHTS AND SLOPES OF FAULT SCARPS OF THE TEPETATE FAULT SYSTEM (SOUTHWEST LOUISIANA)

\begin{tabular}{|c|c|c|c|c|c|}
\hline \multicolumn{2}{|c|}{ Location } & \multirow[b]{2}{*}{ Height (m) } & \multirow[b]{2}{*}{ Slope $(\mathrm{m} / \mathrm{m})$} & \multirow[b]{2}{*}{$\begin{array}{l}\text { Height Error } \\
(\mathrm{m})\end{array}$} & \multirow[b]{2}{*}{$\begin{array}{c}\text { Slope Error } \\
(\mathrm{m})\end{array}$} \\
\hline Longitude & Latitude & & & & \\
\hline-93.38718 & 30.42212 & 2.784 & -0.0204 & 0.023 & 0.00000594 \\
\hline-93.38229 & 30.42254 & 1.643 & -0.0176 & 0.053 & 0.00040660 \\
\hline-93.37987 & 30.42295 & 5.342 & -0.0181 & 0.026 & 0.00000061 \\
\hline-93.37733 & 30.42370 & 9.841 & -0.0388 & 0.074 & 0.00007389 \\
\hline-93.36354 & 30.42113 & 1.426 & -0.0072 & 0.014 & 0.00000008 \\
\hline-93.34522 & 30.41557 & 10.287 & -0.0174 & 0.030 & 0.00106979 \\
\hline-93.34152 & 30.41408 & 7.476 & -0.0253 & 0.075 & 0.00001769 \\
\hline-93.32326 & 30.41923 & 11.204 & -0.0379 & 0.044 & 0.00188959 \\
\hline-93.31139 & 30.42034 & 8.734 & -0.0296 & 0.113 & 0.00113431 \\
\hline-93.29909 & 30.42326 & 8.540 & -0.0385 & 0.126 & 0.00170576 \\
\hline-93.23485 & 30.51643 & 5.169 & -0.0175 & 0.012 & 0.00000078 \\
\hline-93.21961 & 30.51671 & 3.989 & -0.0202 & 0.025 & 0.00005252 \\
\hline-93.21213 & 30.51825 & 7.197 & -0.0162 & 0.101 & 0.00007004 \\
\hline-93.19877 & 30.52087 & 6.702 & -0.0605 & 0.103 & 0.00326621 \\
\hline-93.18857 & 30.52152 & 8.643 & -0.0390 & 0.395 & 0.01106927 \\
\hline-93.17893 & 30.52322 & 5.297 & -0.0179 & 0.154 & 0.00047935 \\
\hline-93.20366 & 30.51943 & 1.077 & -0.0061 & 0.015 & 0.00001087 \\
\hline-93.16014 & 30.52518 & 1.102 & -0.0143 & 0.017 & 0.00000867 \\
\hline-93.12695 & 30.53010 & 5.397 & -0.0304 & 0.053 & 0.00134353 \\
\hline-93.12304 & 30.53003 & 10.469 & -0.0236 & 0.209 & 0.00005461 \\
\hline-93.11923 & 30.53002 & 9.045 & -0.0255 & 0.078 & 0.00000396 \\
\hline-93.11566 & 30.52991 & 7.264 & -0.0205 & 0.100 & 0.00264864 \\
\hline-93.11089 & 30.52944 & 5.463 & -0.0123 & 0.056 & 0.00071758 \\
\hline-93.10618 & 30.52891 & 5.378 & -0.0152 & 0.186 & 0.00482885 \\
\hline-93.08030 & 30.50365 & 4.915 & -0.0222 & 0.106 & 0.00038677 \\
\hline-93.07484 & 30.50717 & 7.204 & -0.0244 & 0.024 & 0.00003117 \\
\hline-93.07221 & 30.50827 & 2.375 & -0.0094 & 0.008 & 0.00000130 \\
\hline-93.06394 & 30.51139 & 1.199 & -0.0034 & 0.005 & 0.00003053 \\
\hline-93.04410 & 30.51748 & 5.427 & -0.0184 & 0.074 & 0.00045996 \\
\hline-93.03173 & 30.52010 & 5.836 & -0.0165 & 0.043 & 0.00018023 \\
\hline-93.02713 & 30.52035 & 4.656 & -0.0158 & 0.014 & 0.00000153 \\
\hline-93.02338 & 30.52163 & 3.404 & -0.0173 & 0.033 & 0.00021038 \\
\hline-93.01989 & 30.52293 & 3.099 & -0.0262 & 0.072 & 0.00000121 \\
\hline-93.01401 & 30.52314 & 2.768 & -0.0125 & 0.029 & 0.00039932 \\
\hline-93.00713 & 30.52344 & 1.666 & -0.0469 & 0.035 & 0.00007449 \\
\hline-92.99976 & 30.52417 & 1.750 & -0.0049 & 0.027 & 0.00000073 \\
\hline-92.96128 & 30.51841 & 1.463 & -0.0190 & 0.019 & 0.00026019 \\
\hline-92.94187 & 30.51524 & 1.343 & -0.0030 & 0.005 & 0.00000924 \\
\hline-93.38627 & 30.51684 & 5.450 & -0.0123 & 0.043 & 0.00014707 \\
\hline-93.33515 & 30.53129 & 6.533 & -0.0332 & 0.184 & 0.00000239 \\
\hline-92.85029 & 30.52195 & 1.909 & -0.0172 & 0.031 & 0.00027528 \\
\hline
\end{tabular}


TABLE S18 CONTINUED. HEIGHTS AND SLOPES OF FAULT SCARPS OF THE TEPETATE FAULT SYSTEM (SOUTHWEST LOUISIANA)

\begin{tabular}{|c|c|c|c|c|c|}
\hline \multicolumn{2}{|c|}{ Location } & \multirow[b]{2}{*}{ Height (m) } & \multirow[b]{2}{*}{ Slope $(\mathrm{m} / \mathrm{m})$} & \multirow[b]{2}{*}{$\begin{array}{l}\text { Height Error } \\
(\mathrm{m})\end{array}$} & \multirow[b]{2}{*}{$\begin{array}{c}\text { Slope Error } \\
(\mathrm{m})\end{array}$} \\
\hline Longitude & Latitude & & & & \\
\hline-92.83958 & 30.52151 & 1.882 & -0.0106 & 0.009 & 0.00000191 \\
\hline-92.82877 & 30.52091 & 1.469 & -0.0364 & 0.053 & 0.00020003 \\
\hline-92.82609 & 30.52034 & 1.563 & -0.0123 & 0.011 & 0.00012025 \\
\hline-92.82341 & 30.51976 & 1.991 & -0.0067 & 0.025 & 0.00054731 \\
\hline-92.82020 & 30.51954 & 1.968 & -0.0067 & 0.058 & 0.00000017 \\
\hline-92.81699 & 30.52148 & 1.357 & -0.0084 & 0.008 & 0.00000103 \\
\hline-92.80798 & 30.52308 & 1.537 & -0.0069 & 0.011 & 0.00016945 \\
\hline-92.80096 & 30.52533 & 2.719 & -0.0199 & 0.018 & 0.00001022 \\
\hline-92.77940 & 30.52736 & 1.481 & -0.0100 & 0.024 & 0.00009701 \\
\hline-92.76989 & 30.52636 & 1.139 & -0.0058 & 0.008 & 0.00003026 \\
\hline-92.76946 & 30.52645 & 1.275 & -0.0058 & 0.008 & 0.00003629 \\
\hline-92.77922 & 30.52726 & 1.579 & -0.0098 & 0.025 & 0.00010742 \\
\hline-92.75543 & 30.52177 & 2.997 & -0.0085 & 0.013 & 0.00001453 \\
\hline-92.75035 & 30.52116 & 3.857 & -0.0131 & 0.057 & 0.00082498 \\
\hline-92.74641 & 30.52100 & 2.748 & -0.0078 & 0.042 & 0.00000044 \\
\hline-92.74115 & 30.52073 & 1.602 & -0.0045 & 0.006 & 0.00009875 \\
\hline-92.73548 & 30.51840 & 2.112 & -0.0083 & 0.043 & 0.00000215 \\
\hline-92.73221 & 30.52019 & 2.265 & -0.0115 & 0.010 & 0.00012209 \\
\hline-92.72410 & 30.52485 & 2.424 & -0.0205 & 0.031 & 0.00010512 \\
\hline-92.71585 & 30.52953 & 4.751 & -0.0214 & 0.018 & 0.00020307 \\
\hline-92.70180 & 30.53578 & 2.167 & -0.0086 & 0.021 & 0.00013987 \\
\hline-92.68820 & 30.53744 & 1.447 & -0.0139 & 0.017 & 0.00032670 \\
\hline-92.64012 & 30.53783 & 1.433 & -0.0650 & 0.010 & 0.00014177 \\
\hline-92.79421 & 30.63224 & 2.7114 & -0.0092 & 0.335 & 0.00003390 \\
\hline-92.78675 & 30.63426 & 3.1074 & -0.0088 & 0.119 & 0.00003828 \\
\hline-92.77905 & 30.63523 & 3.1739 & -0.0089 & 0.017 & 0.00000597 \\
\hline-92.77357 & 30.63648 & 2.1504 & -0.0109 & 0.008 & 0.00013839 \\
\hline-92.76956 & 30.63771 & 1.8334 & -0.0062 & 0.016 & 0.00000863 \\
\hline-92.76083 & 30.63869 & 0.5133 & -0.0026 & 0.002 & 0.00000128 \\
\hline-92.73831 & 30.63691 & 2.8962 & -0.0098 & 0.016 & 0.00000024 \\
\hline-92.72565 & 30.63828 & 2.2274 & -0.0075 & 0.015 & 0.00000565 \\
\hline-92.71350 & 30.63979 & 2.083 & -0.0117 & 0.018 & 0.00002165 \\
\hline-92.70556 & 30.64135 & 1.2577 & -0.0092 & 0.018 & 0.00021080 \\
\hline-92.69894 & 30.64190 & 1.7754 & -0.005 & 0.022 & 0.00009023 \\
\hline-92.99680 & 30.58916 & 2.7646 & -0.0078 & 0.043 & 0.00006859 \\
\hline-92.99354 & 30.58841 & 4.5188 & -0.0102 & 0.044 & 0.00000209 \\
\hline-92.98719 & 30.58843 & 5.0047 & -0.0141 & 0.04 & 0.00000194 \\
\hline-92.97791 & 30.58897 & 2.8791 & -0.0179 & 0.007 & 0.00003396 \\
\hline-92.96711 & 30.59082 & 2.0218 & -0.0182 & 0.005 & 0.00002127 \\
\hline-92.91416 & 30.58339 & 3.5534 & -0.016 & 0.016 & 0.00003593 \\
\hline-92.90061 & 30.58680 & 3.1602 & -0.0089 & 0.027 & 0.00000408 \\
\hline
\end{tabular}


TABLE S18 CONTINUED. HEIGHTS AND SLOPES OF FAULT SCARPS OF THE TEPETATE FAULT SYSTEM (SOUTHWEST LOUISIANA)

\begin{tabular}{lccccc}
\hline \multicolumn{2}{c}{ Location } & & & & \\
\cline { 1 - 2 } Longitude & Latitude & Height $(\mathrm{m})$ & Slope $(\mathrm{m} / \mathrm{m})$ & $\begin{array}{c}\text { Height Error } \\
(\mathrm{m})\end{array}$ & $\begin{array}{c}\text { Slope Error } \\
(\mathrm{m})\end{array}$ \\
\hline-92.89286 & 30.58885 & 1.9278 & -0.0098 & 0.016 & 0.00017195 \\
-92.83124 & 30.59370 & 1.7562 & -0.004 & 0.016 & 0.00003866 \\
-92.79423 & 30.58919 & 1.7054 & -0.087 & 0.016 & 0.00013494 \\
-92.77062 & 30.59074 & 2.7914 & -0.0173 & 0.024 & 0.00022378 \\
-92.76511 & 30.59005 & 1.6024 & -0.0072 & 0.01 & 0.00007536 \\
-92.78554 & 30.59160 & 1.437 & -0.0049 & 0.017 & 0.00000406 \\
-92.75243 & 30.58909 & 1.2813 & -0.0029 & 0.008 & 0.00001063 \\
-92.74830 & 30.58887 & 1.7261 & -0.0058 & 0.008 & 0.00000185
\end{tabular}

TABLE S19. HEIGHTS AND SLOPES OF FAULT SCARPS OF THE BATON ROUGE FAULT SYSTEM (SOUTHEAST LOUISIANA)

\begin{tabular}{|c|c|c|c|c|c|}
\hline \multicolumn{2}{|c|}{ Location } & \multirow[b]{2}{*}{ Height (m) } & \multirow[b]{2}{*}{ Slope $(\mathrm{m} / \mathrm{m})$} & \multirow[b]{2}{*}{$\begin{array}{l}\text { Height Error } \\
(\mathrm{m})\end{array}$} & \multirow[b]{2}{*}{$\begin{array}{c}\text { Slope Error } \\
(\mathrm{m})\end{array}$} \\
\hline Longitude & Latitude & & & & \\
\hline-90.801 & 30.419 & 1.645 & -0.0120 & 0.014 & 0.000378 \\
\hline-90.788 & 30.412 & 4.026 & -0.0240 & 0.159 & 0.001300 \\
\hline-90.770 & 30.415 & 4.104 & -0.0190 & 0.036 & 0.000082 \\
\hline-90.767 & 30.414 & 1.634 & -0.0110 & 0.034 & 0.000952 \\
\hline-90.758 & 30.416 & 2.694 & -0.0080 & 0.045 & 0.000039 \\
\hline-90.753 & 30.416 & 2.303 & -0.0080 & 0.023 & 0.000000 \\
\hline-90.736 & 30.416 & 1.965 & -0.0180 & 0.012 & 0.000057 \\
\hline-90.733 & 30.416 & 1.667 & -0.0180 & 0.016 & 0.000140 \\
\hline-90.722 & 30.415 & 1.307 & -0.0250 & 0.019 & 0.000043 \\
\hline-90.719 & 30.414 & 1.042 & -0.0080 & 0.026 & 0.000045 \\
\hline-90.713 & 30.412 & 1.289 & -0.0140 & 0.048 & 0.000124 \\
\hline-90.709 & 30.411 & 1.094 & -0.0310 & 0.017 & 0.000066 \\
\hline-90.617 & 30.404 & 1.253 & -0.0260 & 0.034 & 0.000027 \\
\hline-90.609 & 30.404 & 0.652 & -0.0060 & 0.026 & 0.000323 \\
\hline-90.600 & 30.405 & 1.318 & -0.0060 & 0.017 & 0.000008 \\
\hline-90.596 & 30.405 & 1.599 & -0.0110 & 0.049 & 0.000078 \\
\hline-90.589 & 30.406 & 2.262 & -0.0150 & 0.029 & 0.000020 \\
\hline-90.584 & 30.406 & 1.479 & -0.0420 & 0.032 & 0.000032 \\
\hline-90.579 & 30.406 & 2.638 & -0.0230 & 0.031 & 0.000258 \\
\hline-90.573 & 30.406 & 1.601 & -0.0300 & 0.047 & 0.000315 \\
\hline-90.565 & 30.407 & 0.891 & -0.0250 & 0.014 & 0.000052 \\
\hline-90.553 & 30.407 & 1.825 & -0.0090 & 0.018 & 0.000008 \\
\hline-90.544 & 30.416 & 2.041 & -0.0040 & 0.018 & 0.000118 \\
\hline-90.542 & 30.416 & 1.631 & -0.0200 & 0.013 & 0.000013 \\
\hline
\end{tabular}


TABLE S19 CONTINUED. HEIGHTS AND SLOPES OF FAULT SCARPS OF THE BATON ROUGE FAULT SYSTEM (SOUTHEAST LOUISIANA)

\begin{tabular}{|c|c|c|c|c|c|}
\hline \multicolumn{2}{|c|}{ Location } & \multirow[b]{2}{*}{ Height (m) } & \multirow[b]{2}{*}{ Slope $(\mathrm{m} / \mathrm{m})$} & \multirow[b]{2}{*}{$\begin{array}{l}\text { Height Error } \\
(\mathrm{m})\end{array}$} & \multirow[b]{2}{*}{$\begin{array}{c}\text { Slope Error } \\
(\mathrm{m})\end{array}$} \\
\hline Longitude & Latitude & & & & \\
\hline-90.540 & 30.416 & 1.862 & -0.0080 & 0.013 & 0.000251 \\
\hline-90.538 & 30.416 & 1.403 & -0.0400 & 0.061 & 0.000042 \\
\hline-90.534 & 30.416 & 1.997 & -0.0070 & 0.089 & 0.001060 \\
\hline-90.412 & 30.414 & 2.292 & -0.0650 & 0.079 & 0.000369 \\
\hline-90.409 & 30.414 & 2.534 & -0.0590 & 0.051 & 0.000214 \\
\hline-90.405 & 30.414 & 2.642 & -0.0540 & 0.041 & 0.000535 \\
\hline-90.402 & 30.414 & 3.348 & -0.0290 & 0.047 & 0.000058 \\
\hline-90.403 & 30.414 & 3.05 & -0.0380 & 0.034 & 0.000348 \\
\hline-90.345 & 30.408 & 1.357 & -0.0330 & 0.014 & 0.000027 \\
\hline-90.351 & 30.409 & 0.835 & -0.0240 & 0.01 & 0.000016 \\
\hline-90.358 & 30.410 & 2.151 & -0.0260 & 0.02 & 0.000085 \\
\hline-90.371 & 30.412 & 2.179 & -0.0610 & 0.171 & 0.001261 \\
\hline-90.332 & 30.412 & 1.312 & -0.0370 & 0.029 & 0.000041 \\
\hline-90.324 & 30.409 & 1.132 & -0.0300 & 0.047 & 0.000374 \\
\hline-90.322 & 30.404 & 0.995 & -0.0280 & 0.015 & 0.000000 \\
\hline-90.319 & 30.404 & 1.139 & -0.0140 & 0.01 & 0.000053 \\
\hline-90.317 & 30.404 & 0.675 & -0.0060 & 0.03 & 0.000483 \\
\hline-90.302 & 30.405 & 0.789 & -0.0020 & 0.005 & 0.000076 \\
\hline-90.291 & 30.405 & 1.169 & -0.0030 & 0.008 & 0.000032 \\
\hline-90.270 & 30.400 & 0.48 & -0.0140 & 0.007 & 0.000002 \\
\hline-90.276 & 30.403 & 1.204 & -0.0050 & 0.008 & 0.000008 \\
\hline-90.284 & 30.404 & 1.148 & -0.0040 & 0.009 & 0.000040 \\
\hline-90.538 & 30.416 & 1.403 & -0.0400 & 0.061 & 0.000042 \\
\hline-90.534 & 30.416 & 1.997 & -0.0070 & 0.089 & 0.001060 \\
\hline-90.505 & 30.421 & 1.789 & -0.0290 & 0.042 & 0.000008 \\
\hline-90.496 & 30.422 & 2.928 & -0.0400 & 0.054 & 0.000156 \\
\hline-90.488 & 30.423 & 3.73 & -0.0310 & 0.114 & 0.000875 \\
\hline-90.483 & 30.424 & 3.418 & -0.0400 & 0.124 & 0.001786 \\
\hline-90.506 & 30.421 & 2.313 & -0.0390 & 0.135 & 0.003505 \\
\hline-90.501 & 30.422 & 2.823 & -0.0390 & 0.041 & 0.000038 \\
\hline-90.496 & 30.422 & 2.6 & -0.0430 & 0.202 & 0.000031 \\
\hline-90.488 & 30.423 & 3.393 & -0.0370 & 0.14 & 0.000498 \\
\hline-90.484 & 30.424 & 3.516 & -0.0490 & 0.162 & 0.000556 \\
\hline-90.467 & 30.424 & 2.642 & -0.0480 & 0.023 & 0.000013 \\
\hline-90.463 & 30.424 & 2.853 & -0.0400 & 0.017 & 0.000000 \\
\hline-90.459 & 30.423 & 2.931 & -0.0250 & 0.02 & 0.000068 \\
\hline-90.455 & 30.423 & 2.826 & -0.0150 & 0.018 & 0.000176 \\
\hline-90.453 & 30.422 & 2.597 & -0.0250 & 0.034 & 0.000094 \\
\hline-90.451 & 30.422 & 2.375 & -0.0220 & 0.021 & 0.000653 \\
\hline-90.444 & 30.421 & 2.752 & -0.0100 & 0.169 & 0.000020 \\
\hline-90.443 & 30.420 & 2.336 & -0.0250 & 0.179 & 0.000446 \\
\hline
\end{tabular}


TABLE S19 CONTINUED. HEIGHTS AND SLOPES OF FAULT SCARPS OF THE BATON ROUGE FAULT SYSTEM (SOUTHEAST LOUISIANA)

\begin{tabular}{|c|c|c|c|c|c|}
\hline \multicolumn{2}{|c|}{ Location } & \multirow[b]{2}{*}{ Height (m) } & \multirow[b]{2}{*}{ Slope $(\mathrm{m} / \mathrm{m})$} & \multirow[b]{2}{*}{$\begin{array}{l}\text { Height Error } \\
(\mathrm{m})\end{array}$} & \multirow[b]{2}{*}{$\begin{array}{c}\text { Slope Error } \\
(\mathrm{m})\end{array}$} \\
\hline Longitude & Latitude & & & & \\
\hline-90.441 & 30.420 & 2.693 & -0.0310 & 0.186 & 0.000703 \\
\hline-90.439 & 30.419 & 2.668 & -0.0460 & 0.112 & 0.000772 \\
\hline-90.438 & 30.420 & 2.645 & -0.0130 & 0.122 & 0.000847 \\
\hline-90.437 & 30.419 & 2.193 & -0.0150 & 0.152 & 0.000032 \\
\hline-90.672 & 30.428 & 1.53 & -0.0100 & 0.046 & 0.000169 \\
\hline-90.661 & 30.427 & 1.632 & -0.0170 & 0.089 & 0.000028 \\
\hline-90.667 & 30.428 & 2.117 & -0.0100 & 0.041 & 0.000297 \\
\hline-90.941 & 30.394 & 4.997 & -0.0510 & 0.084 & 0.000180 \\
\hline-90.932 & 30.394 & 5.969 & -0.0480 & 0.101 & 0.000127 \\
\hline-90.925 & 30.396 & 4.118 & -0.0460 & 0.067 & 0.000036 \\
\hline-90.910 & 30.393 & 1.261 & -0.0360 & 0.054 & 0.000003 \\
\hline-90.897 & 30.391 & 1.858 & -0.0520 & 0.074 & 0.000001 \\
\hline-90.884 & 30.391 & 1.326 & -0.0370 & 0.146 & 0.000962 \\
\hline-91.101 & 30.419 & 4.219 & -0.0480 & 0.403 & 0.004854 \\
\hline-91.083 & 30.414 & 3.983 & -0.0390 & 0.126 & 0.000055 \\
\hline-91.087 & 30.414 & 4.249 & -0.0510 & 0.26 & 0.001287 \\
\hline-91.081 & 30.413 & 4.358 & -0.0260 & 0.052 & 0.000030 \\
\hline-91.078 & 30.411 & 5.1 & -0.0560 & 0.045 & 0.000994 \\
\hline-91.073 & 30.410 & 5.217 & -0.0650 & 0.127 & 0.002596 \\
\hline-91.065 & 30.408 & 5.047 & -0.0650 & 0.333 & 0.000943 \\
\hline-91.056 & 30.403 & 3.945 & -0.0220 & 0.234 & 0.000000 \\
\hline-91.050 & 30.396 & 4.548 & -0.0510 & 0.464 & 0.000016 \\
\hline-91.043 & 30.395 & 4.473 & -0.0490 & 0.355 & 0.000106 \\
\hline-91.035 & 30.393 & 4.203 & -0.0190 & 0.29 & 0.000087 \\
\hline-91.030 & 30.393 & 4.232 & -0.0420 & 0.518 & 0.008424 \\
\hline-91.023 & 30.392 & 5.139 & -0.0300 & 0.397 & 0.001236 \\
\hline-91.016 & 30.392 & 4.447 & -0.0460 & 0.323 & 0.000595 \\
\hline-91.003 & 30.389 & 5.328 & -0.0670 & 0.623 & 0.004489 \\
\hline-90.832 & 30.493 & 1.288 & -0.0280 & 0.021 & 0.000329 \\
\hline-90.820 & 30.493 & 1.112 & -0.0230 & 0.034 & 0.000002 \\
\hline-90.809 & 30.493 & 1.053 & -0.0280 & 0.017 & 0.000057 \\
\hline-90.815 & 30.493 & 1.884 & -0.0240 & 0.034 & 0.000310 \\
\hline-90.975 & 30.389 & 5.321 & -0.0680 & 0.078 & 0.000020 \\
\hline-90.984 & 30.389 & 5.073 & -0.0560 & 0.449 & 0.000020 \\
\hline-90.960 & 30.481 & 2.66 & -0.0220 & 0.029 & 0.000270 \\
\hline-90.955 & 30.479 & 2.712 & -0.0260 & 0.043 & 0.000049 \\
\hline-90.953 & 30.479 & 2.744 & -0.0400 & 0.069 & 0.000108 \\
\hline-90.951 & 30.478 & 3.05 & -0.0200 & 0.033 & 0.000032 \\
\hline-90.948 & 30.478 & 3.365 & -0.0270 & 0.035 & 0.000274 \\
\hline-90.944 & 30.479 & 3.08 & -0.0210 & 0.015 & 0.000303 \\
\hline-90.936 & 30.480 & 3.294 & -0.0170 & 0.09 & 0.000579 \\
\hline
\end{tabular}


TABLE S19 CONTINUED. HEIGHTS AND SLOPES OF FAULT SCARPS OF THE BATON ROUGE FAULT SYSTEM (SOUTHEAST LOUISIANA)

\begin{tabular}{|c|c|c|c|c|c|}
\hline \multicolumn{2}{|c|}{ Location } & \multirow[b]{2}{*}{ Height (m) } & \multirow[b]{2}{*}{ Slope $(\mathrm{m} / \mathrm{m})$} & \multirow[b]{2}{*}{$\begin{array}{l}\text { Height Error } \\
(\mathrm{m})\end{array}$} & \multirow[b]{2}{*}{$\begin{array}{c}\text { Slope Error } \\
(\mathrm{m})\end{array}$} \\
\hline Longitude & Latitude & & & & \\
\hline-90.932 & 30.482 & 3.357 & -0.0120 & 0.027 & 0.000264 \\
\hline-90.927 & 30.482 & 2.085 & -0.0110 & 0.031 & 0.000228 \\
\hline-90.923 & 30.482 & 1.819 & -0.0190 & 0.03 & 0.000020 \\
\hline-91.148 & 30.429 & 7.793 & -0.0310 & 0.105 & 0.001148 \\
\hline-91.144 & 30.429 & 5.132 & -0.0470 & 0.078 & 0.000516 \\
\hline-91.139 & 30.428 & 6.332 & -0.0790 & 0.326 & 0.000107 \\
\hline-91.134 & 30.427 & 5.676 & -0.0370 & 0.078 & 0.001213 \\
\hline-91.131 & 30.426 & 4.059 & -0.0390 & 0.04 & 0.000044 \\
\hline-91.122 & 30.426 & 5.821 & -0.0130 & 0.157 & 0.000027 \\
\hline-91.117 & 30.423 & 5.89 & -0.0530 & 0.092 & 0.000017 \\
\hline-91.114 & 30.422 & 4.87 & -0.0440 & 0.226 & 0.000574 \\
\hline-91.108 & 30.421 & 3.863 & -0.0570 & 0.165 & 0.000823 \\
\hline-91.101 & 30.419 & 4.219 & -0.0480 & 0.403 & 0.004854 \\
\hline-91.083 & 30.414 & 3.983 & -0.0390 & 0.126 & 0.000055 \\
\hline-91.087 & 30.414 & 4.249 & -0.0510 & 0.26 & 0.001287 \\
\hline-91.081 & 30.413 & 4.358 & -0.0260 & 0.052 & 0.000030 \\
\hline-91.078 & 30.411 & 5.1 & -0.0560 & 0.045 & 0.000994 \\
\hline-91.073 & 30.410 & 5.217 & -0.0650 & 0.127 & 0.002596 \\
\hline-91.065 & 30.408 & 5.047 & -0.0650 & 0.333 & 0.000943 \\
\hline-91.056 & 30.403 & 3.945 & -0.0220 & 0.234 & 0.000000 \\
\hline-91.050 & 30.396 & 4.548 & -0.0510 & 0.464 & 0.000016 \\
\hline-91.043 & 30.395 & 4.473 & -0.0490 & 0.355 & 0.000106 \\
\hline-91.035 & 30.393 & 4.203 & -0.0190 & 0.29 & 0.000087 \\
\hline-91.030 & 30.393 & 4.232 & -0.0420 & 0.518 & 0.008424 \\
\hline-91.023 & 30.392 & 5.139 & -0.0300 & 0.397 & 0.001236 \\
\hline-91.016 & 30.392 & 4.447 & -0.0460 & 0.323 & 0.000595 \\
\hline-91.003 & 30.389 & 5.328 & -0.0670 & 0.623 & 0.004489 \\
\hline-91.152 & 30.514 & 2.322 & -0.021 & 0.036 & 0.00001903 \\
\hline-91.114 & 30.51 & 1.299 & -0.034 & 0.134 & 0.00026322 \\
\hline-91.133 & 30.514 & 1.661 & -0.024 & 0.103 & 0.00002542 \\
\hline-91.092 & 30.514 & 2.416 & -0.01 & 0.064 & 0.00009418 \\
\hline-91.088 & 30.514 & 2.921 & -0.012 & 0.22 & 0.00047946 \\
\hline-91.071 & 30.5135 & 4.188 & -0.017 & 0.188 & 0.0000546 \\
\hline-91.066 & 30.513 & 2.686 & -0.037 & 0.087 & 0.00049585 \\
\hline-91.063 & 30.512 & 2.653 & -0.066 & 0.209 & 0.00015964 \\
\hline-91.013 & 30.501 & 0.789 & -0.022 & 0.051 & 0.00095166 \\
\hline-90.984 & 30.489 & 4.017 & -0.05 & 0.212 & 0.00003418 \\
\hline-90.99 & 30.492 & 3.576 & -0.028 & 0.235 & 0.00030533 \\
\hline-90.96 & 30.481 & 2.66 & -0.022 & 0.029 & 0.00027048 \\
\hline-90.955 & 30.479 & 2.712 & -0.026 & 0.043 & 0.00004908 \\
\hline-90.953 & 30.479 & 2.744 & -0.04 & 0.069 & 0.00010802 \\
\hline
\end{tabular}


TABLE S19 CONTINUED. HEIGHTS AND SLOPES OF FAULT SCARPS OF THE BATON ROUGE FAULT SYSTEM (SOUTHEAST LOUISIANA)

\begin{tabular}{|c|c|c|c|c|c|}
\hline \multicolumn{2}{|c|}{ Location } & \multirow[b]{2}{*}{ Height (m) } & \multirow[b]{2}{*}{ Slope $(\mathrm{m} / \mathrm{m})$} & \multirow[b]{2}{*}{$\begin{array}{l}\text { Height Error } \\
(\mathrm{m})\end{array}$} & \multirow[b]{2}{*}{$\begin{array}{l}\text { Slope Error } \\
(\mathrm{m})\end{array}$} \\
\hline Longitude & Latitude & & & & \\
\hline-90.948 & 30.478 & 3.365 & -0.027 & 0.035 & 0.00027433 \\
\hline-90.944 & 30.479 & 3.08 & -0.021 & 0.015 & 0.00030317 \\
\hline-90.936 & 30.48 & 3.294 & -0.017 & 0.09 & 0.00057877 \\
\hline-90.932 & 30.482 & 3.357 & -0.012 & 0.027 & 0.00026425 \\
\hline-90.927 & 30.482 & 2.085 & -0.011 & 0.031 & 0.00022785 \\
\hline-90.923 & 30.482 & 1.819 & -0.019 & 0.03 & 0.00001971 \\
\hline-90.911 & 30.483 & 2.317 & -0.007 & 0.035 & 0.00017096 \\
\hline-90.906 & 30.483 & 2.123 & -0.015 & 0.045 & 0.00002833 \\
\hline-90.901 & 30.483 & 2.061 & -0.01 & 0.028 & 0.00003275 \\
\hline-90.896 & 30.483 & 1.561 & -0.017 & 0.027 & 0.00005151 \\
\hline-90.891 & 30.483 & 1.893 & -0.014 & 0.062 & 0.00064292 \\
\hline-90.886 & 30.483 & 2.028 & -0.006 & 0.034 & 0.00011322 \\
\hline-90.878 & 30.483 & 3.238 & -0.024 & 0.023 & 0.00000265 \\
\hline-90.872 & 30.482 & 2.836 & -0.021 & 0.106 & 0.00009925 \\
\hline-90.867 & 30.481 & 2.278 & -0.019 & 0.058 & 0.00073726 \\
\hline-90.862 & 30.48 & 2.838 & -0.017 & 0.016 & 0.00000122 \\
\hline-90.846 & 30.478 & 2.273 & -0.012 & 0.031 & 0.00003926 \\
\hline-90.853 & 30.478 & 2.227 & -0.025 & 0.058 & 0.00001825 \\
\hline-90.832 & 30.493 & 1.288 & -0.028 & 0.021 & 0.00032921 \\
\hline-90.82 & 30.493 & 1.112 & -0.023 & 0.034 & 0.0000022 \\
\hline-90.809 & 30.493 & 1.053 & -0.028 & 0.017 & 0.00005673 \\
\hline-90.815 & 30.493 & 1.884 & -0.024 & 0.034 & 0.00031048 \\
\hline-90.796 & 30.488 & 2.568 & -0.014 & 0.049 & 0.00011198 \\
\hline-90.792 & 30.488 & 2.266 & -0.017 & 0.029 & 0.00016351 \\
\hline-90.787 & 30.489 & 1.632 & -0.023 & 0.06 & 0.00002865 \\
\hline-90.778 & 30.489 & 1.427 & -0.016 & 0.035 & 0.00002848 \\
\hline-90.768 & 30.489 & 1.478 & -0.009 & 0.014 & 0.00000035 \\
\hline-90.765 & 30.488 & 1.648 & -0.017 & 0.021 & 0.00000008 \\
\hline-90.76 & 30.487 & 1.631 & -0.017 & 0.028 & 0.00001422 \\
\hline-90.756 & 30.485 & 1.331 & -0.032 & 0.032 & 0.00000875 \\
\hline-90.747 & 30.487 & 1.485 & -0.007 & 0.052 & 0.00064227 \\
\hline-90.656 & 30.48 & 1.16 & -0.033 & 0.035 & 0.00001192 \\
\hline-90.691 & 30.48 & 1.426 & -0.005 & 0.047 & 0.00014119 \\
\hline-90.668 & 30.472 & 3.441 & -0.027 & 0.077 & 0.00040219 \\
\hline-90.665 & 30.472 & 3.624 & -0.024 & 0.057 & 0.00016088 \\
\hline-90.653 & 30.47 & 2.579 & -0.027 & 0.053 & 0.00026794 \\
\hline-90.661 & 30.471 & 3.298 & -0.023 & 0.093 & 0.00082336 \\
\hline
\end{tabular}

